\section{Cyril FLEURANT 1 , Gregory TUCKER 2 et Heather VILES 3 \\ (1) Département Paysage, Institut national d'horticulture, 2, rue Le Nôtre, 49045 Angers cedex, France. cyril.fleurant@inh.fr \\ (2) Department of Geological Sciences, Cooperative Institute for Research in Environmental Sciences, University of Colorado, Boulder, USA \\ (3) School of Geography and the Environment, University of Oxford, Oxford, UK}

\title{
Modèle d'évolution de paysages, application aux karsts en cockpit de Jamaïque
}

RÉSUMÉ : Cet article présente les résultats d'un modèle géomorphologique d'évolution des karsts en cockpit (Jamaïque). Dans un premier temps, nous expliquons comment les processus de dissolution des carbonates ont été implémentés dans le logiciel d'évolution géomorphologique CHILD. Puis nous donnons les détails de notre modèle d'érosion, basé sur les principes de la théorie de l'épikarst. Le modèle prend en compte l'anisotropie spatio-temporelle conformément à ce qui est observé empiriquement ou encore décrit par des scénarii de l'évolution des karsts en cockpit. Ce modèle nécessite d'abord d'introduire un réseau de fractures afin de prendre en compte les écoulements de sub-surface. Ensuite, la dissolution et donc l'ouverture des fractures sont calculées ce qui participe à un processus de feed-back positif entre dissolution et écoulement. La relation entre la dissolution des carbonates fracturés et l'évolution de la topographie est introduite par le biais d'une relation empirique illustrant les processus de l'approche de l'épikarst. Les simulations des paysages par ce modèle anisotrope donnent des résultats très proches de la réalité et confortent à la fois l'importance de la prise en compte de l'anisotropie spatio-temporelle de la dissolution et surtout la validité de l'approche empirique de l'épikarst pour décrire la genèse des karsts en cockpit. MOTS-CLÉs: érosion, dissolution, karst, fracture, paysage, modélisation.

ABSTRACT: LANDSCAPE EVOLUTION MODEL, EXAMPLE OF COCKPIT KARST TERRAINS, JAMAICA. A model of cockpit karst landscape evolution is presented. After explaining implementation of dissolution processes of limestone in the landscape evolution model CHILD, we develop a model of limestone denudation based on epikarst theory processes. The model takes into account an anisotropic dissolution in space and time according to what is observed in reality or described by scenarios of cockpit karst landscape evolution. This model requires a fracture's network to take into account subsurface flow. Then, dissolution and thus fractures widening are computed and show a positive feedback between dissolution and flow. The relation between subcutaneous dissolution of fractures and denudation of the topography is introduced by means of an empirical equation associated with epikarst processes: the denudation is taken to be proportional to the dissolution in the subcutaneous zone. Simulated cockpit karst terrains are compared with real landscapes by means of morphometric criteria. Results of the model are very close to reality which hence confirms the importance of anisotropic dissolution processes and above all could be a numerical validation of the epikarst processes to describe cockpit karst genesis.

KEY WORDS: erosion, dissolution, karst, fracture, landscape, modelling.

\section{Introduction}

L'évolution des paysages résulte de forces: la tectonique, l'érosion et la sédimentation qui, elles-mêmes, sont le produit de processus complexes dépendants des mouvements lithosphériques, des conditions climatiques ou encore des caractéristiques des roches. La compréhension de l'évolution des paysages demande donc des outils numériques capables de tenir compte de la complexité de ces processus nonlinéaires et interagissant qui façonnent les paysages. Un effort tout particulier a été fourni ces dix dernières années pour mettre en place de tels modèles, capables de simuler l'évolution des paysages sur de grandes échelles de temps. Les principaux modèles généralement utilisés sont passés en revue dans Coulthard [2001]. Il s'agit de SIBERIA [Willgoose et al., 1991], GOLEM [Tucker and Slingerland, 1994], CASCADE [Braun and Sambridge, 1997], CAESAR [Coulthard et al., 1998] et CHILD [Tucker et al., 1999].

Il existe une grande variété de paysages karstiques dont les formes sont contrôlées par de nombreuses variables géologiques, chimiques ou encore climatiques [White, 1984]. Les variations de chacune de ces composantes pourront conduire à un paysage différent: un karst à dolines, un fluviokarst ou encore un karst à tourelles.

Les karsts en cockpits (figure 1) sont décrits comme irréguliers et formés de dépressions en forme d'étoile, ils sont propres aux régions tropicales [Sweeting, 1972]. Grund [1914] avait déjà proposé une hypothèse quant à leur formation: les dépressions des cockpits seraient un état avancé des dolines, leur processus de formation serait donc similaire. 


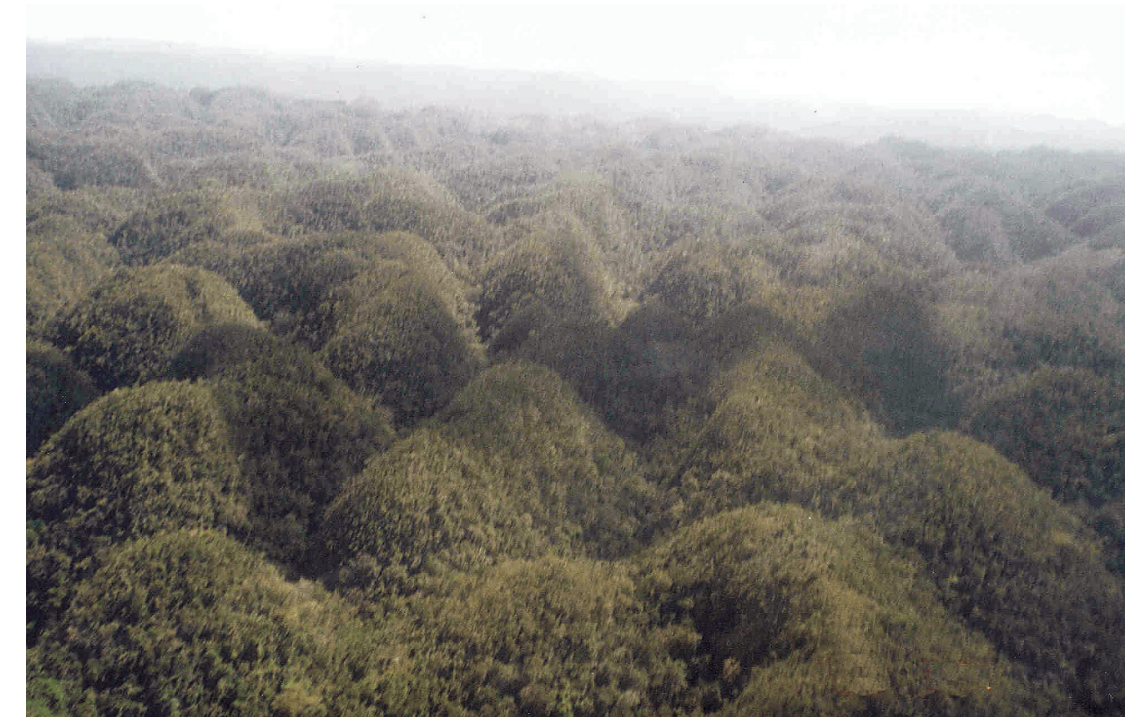

Figure 1: Vue aérienne d'un karst en cockpit en Jamaïque. Cliché de Parris Lyew-Aye.

An oblique aerial view of cockpit karst terrain in Jamaica.

Figure 2: Illustration schématique de

la spatialisation de CHILD [d'après Tucker et al., 2001a]. Schematic illustration of the spatial framework in CHILD.
On ne trouve des karsts en cockpit que dans les régions humides très fortement arrosées par des pluies. Seules des régions dont la quantité de pluie brute est supérieure à $1500 \mathrm{~mm}$ par an sont susceptibles de pouvoir développer ce type de karst [Verstappen, 1964; Sweeting, 1972]. Toutefois, la pluie ne suffit pas, il faut également une roche carbonatée très dure et compacte avec un réseau de fractures préexistant.

On qualifie les processus karstiques par la prédominance du transfert de masse en solution sur le transfert par d'autres processus [White, 1984]. Voilà pourquoi l'intégration des processus de dissolution des roches carbonatées est essentielle pour modéliser les phénomènes karstiques.

Cet article décrit la mise en équation, c'est-à-dire la modélisation, des processus complexes qui illustrent le fonctionnement de l'épikarst dans le cas très particulier des karsts en cockpit. Les processus souterrains jouentils un rôle dans le développement superficiel des karsts en cockpit? Plusieurs auteurs [e.g. Williams, 1987; Pfeffer, 1997] apportent des réponses, il est vrai fondamentales, mais relativement descriptives, à cette problématique.

Les auteurs de cette note n'ont pas la prétention de donner l'ensemble des clés du développement des paysages de karst en cockpit, mais simplement d'apporter un éclairage quantitatif (i.e. par la modélisation

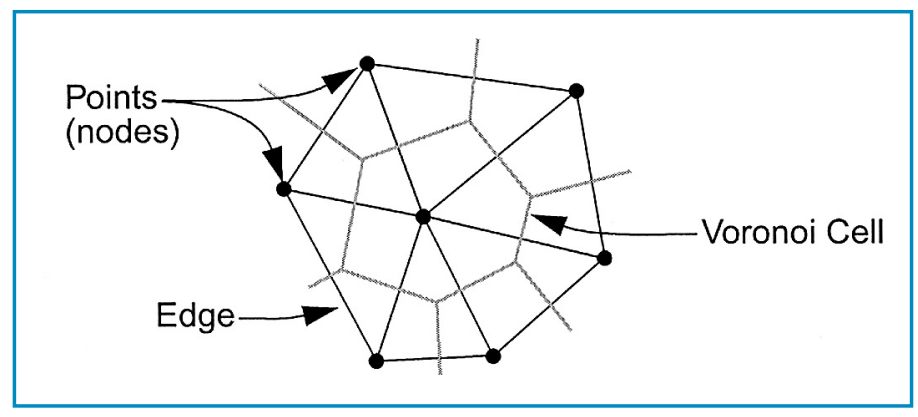

numérique) à cette problématique. L'objectif de cet article est donc de tester des hypothèses relatives à l'importance du rôle souterrain (i.e. de la zone épikarstique) des karsts en cockpit au regard de leur développement géomorphologique. De cette étude, les auteurs dégagent aussi les conditions hydrologiques et géochimiques qui permettent à des paysages karstiques de se développer en cockpit.

Dans un premier temps, nous présenterons l'intégration de processus de dissolution chimique des roches carbonatées dans le modèle CHILD [Tucker et al., 2001a, b]. Puis nous présenterons un modèle de dissolution anisotrope dans l'espace et le temps. Ce modèle sera testé à l'échelle d'un seul cockpit.

Les paysages simulés seront comparés aux cockpits réels par le biais de deux critères géométriques caractéristiques à ce type de paysage et récemment mis en évidence par Lyew-Ayee [2004] : il s'agit de la pente et du relief différentiel (différence entre la hauteur du sommet et la profondeur d'une dépression). Cette comparaison permettra à la fois de donner l'ordre de grandeur des différents paramètres du modèle de dissolution et également d'émettre des hypothèses de scénario sur la formation de ces cockpits.

\section{Le modèle géomorphologique}

Le modèle CHILD [Channel-Hillslope Integrated Landscape Development] est un modèle numérique d'évolution des paysages [Tucker et al., 2001a,b]. L'évolution de la topographie dans le temps est simulée par l'interaction et le feed-back entre les écoulements de surface, l'érosion de la roche et le transport des sédiments. CHILD est un support numérique capable de modéliser un grand nombre de processus liés à la géomorphologie d'un bassin versant, aussi nous ne présenterons ici qu'une petite partie de ses caractéristiques. Dans CHILD, l'évolution de la topographie en fonction du temps est la somme de plusieurs contributions:

$$
\frac{d z}{d t}=\left.\frac{d z}{d t}\right|_{\text {diffusion }}+\left.\frac{d z}{d t}\right|_{\text {erosion }}+\left.\frac{d z}{d t}\right|_{\text {tectonique }}+\left.\frac{d z}{d t}\right|_{\text {dissolution }} ^{\text {(1) }}
$$

Ces différents termes sont résolus numériquement sur le cadre spatial particulier des polygones de Voronoï. Les trois premiers termes sont présents dans la quasitotalité des modèles d'évolution des paysages. Le dernier terme, concernant la dissolution des roches, est l'apport original de ce travail.

Le cadre spatial de CHILD est composé d'une série de points aléatoirement distribués qui permettent une discrétisation fidèle de la surface topographique initiale (figure 2). Ces points, ou nœuds, sont reliés les uns aux 
autres pour former un réseau de triangles irréguliers [Braun and Sambridge, 1997; Tucker et al., 2001a]. Ce maillage est construit suivant les règles de Delaunay, qui offrent de nombreux avantages à la modélisation [Tucker et al., 2001b]. Du point de vue numérique, cette triangulation permet de résoudre des équations aux dérivées partielles en utilisant la méthode des volumes finis [Versteeg and Malalasekera, 1995].

La discrétisation temporelle programmée dans le logiciel CHILD est originale et permet de tenir compte des grandes disparités de temps liées aux processus géomorphologiques: l'évolution de la topographie se fait à l'échelle du millier d'années alors que l'échelle de temps d'une pluie ne sera que de quelques minutes à quelques jours. La variabilité à l'échelle géologique est simulée par une méthode stochastique (loi de Poisson) qui donne la distribution de l'amplitude ( $\bar{P}$ en $\mathrm{m} / \mathrm{an}$ ) et de la fréquence $\left(\frac{\overline{T_{r}}}{\bar{T}_{r}+\bar{T}_{b}}\right)$ des pluies [Eagleason, 1978]. La durée de la pluie est $\bar{T}_{r}$ ans et la période entre deux pluies est $\overline{T_{b}}$ ans. L'inverse de la fréquence, $\frac{\overline{T_{r}}+\overline{T_{b}}}{\bar{T}_{r}}$, est appelé facteur de variabilité climatique et sera utilisé ultérieurement dans les simulations [Tucker and Bras, 2000].

Les écoulements se font des nœuds amont vers les nœuds aval (figure 3), en suivant une procédure simple. Une partie de l'eau totale $\left(Q_{\text {in }} \mathrm{m}^{3} / \mathrm{s}\right)$ qui entre dans le polygone de surface $A_{i} \mathrm{~m}^{2}$ associé au nœud $i$, s'infiltre $\left(Q_{\text {sub }} \mathrm{m}^{3} / \mathrm{s}\right)$. Cette quantité, $Q_{\text {subcapacity }}\left(\mathrm{m}^{3} / \mathrm{s}\right)$, est déterminée par la capacité d'infiltration du sol et de la roche. Le reste de l'eau qui ne s'infiltre pas constitue le ruissellement de surface, $Q_{\text {sur }}\left(\mathrm{m}^{3} / \mathrm{s}\right)$.

Le transport des sédiments par des processus continus sur le versant est modélisé dans CHILD par l'équation classique de diffusion des versants [e.g. Braun and Sambridge, 1997] :

$$
\left.\frac{d z}{d t}\right|_{\text {diffusion }}=k_{D} \Delta^{2} z
$$

où $k_{D}\left(\mathrm{~m}^{2} / \mathrm{s}\right)$ est le coefficient de diffusion du transport des sédiments et $\Delta^{2}$ représente le Laplacien. Beaumont et al. [1992], explicitent $k_{D}$ comme une

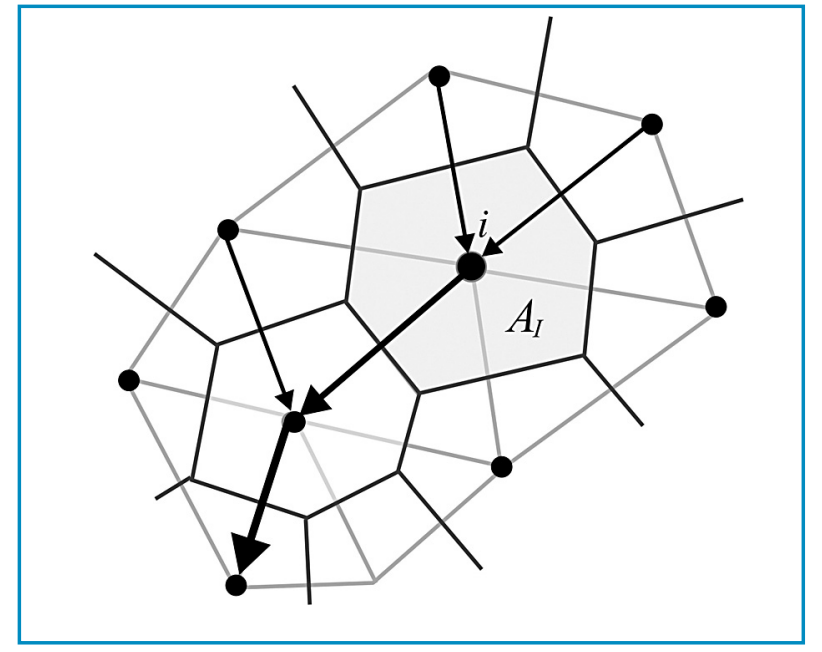

Figure 3: Illustration de la procédure des écoulements sur un maillage triangulaire de Delaunay.

Cette triangulation permet de définir des polygones de surface $A_{i}\left(\mathrm{~m}^{2}\right)$ qui sont les cellules élémentaires du calcul.

Illustration of steepestdescent flow routing in Delaunay TIN framework. TIN framework allows the definition of elementary cells $A_{i}\left(m^{2}\right)$ needed to compute processes.

fonction à la fois de la lithologie $\left(k_{D}\right.$ est faible pour des roches résistantes) et $\mathrm{du}$ climat $\left(\mathrm{k}_{D}\right.$ est plus fort sous des climats plus "rudes"). Ce coefficient de diffusion des sédiments peut varier de $3 \times 10^{-4} \mathrm{~m}^{2} / \mathrm{s}$ [Enzel et al., 1996] à $3 \times 10^{-2} \mathrm{~m}^{2} / \mathrm{s}$ [McKean et al., 1993].

L'érosion mécanique et le dépôt sédimentaire sont modélisés par l'équation de continuité [Tucker et al., 2001a] :

$$
\left.\frac{d z}{d t}\right|_{\text {érosion }}=-\left(\frac{\partial q_{s x}}{\partial x}+\frac{\partial q_{s y}}{\partial y}\right)
$$

où $q_{s}$ est le volume transporté de sédiments.

\section{L'érosion karstique}

Le dioxyde de carbone $\left(\mathrm{CO}_{2}\right)$ présent dans l'atmosphère et produit par la végétation se retrouve sous forme dissoute dans l'eau de pluie et dans les sols. La forme dissoute du $\mathrm{CO}_{2}$, l'acide carbonique $\mathrm{H}_{2} \mathrm{CO}_{3}$, est l'agent majeur de l'érosion des roches carbonatées.

L'évolution spatiale et temporelle des systèmes karstiques dépend de la cinétique de la dissolution des carbonates, et plus précisément de la calcite $\left(\mathrm{CaCO}_{3}\right)$. On distingue le système ouvert où les carbonates sont en contact avec les intempéries atmosphériques par opposition au système fermé, cas des fractures par exemple, qui sont très peu en contact avec l'extérieur. Dans le cas du système ouvert, la dissolution des roches carbonatées va alors dépendre de la hauteur d'eau en contact avec les carbonates et de la nature hydrodynamique du flux [Dreybrodt, 1985].

La vitesse de l'érosion karstique correspond principalement à la vitesse d'érosion des carbonates par dissolution. Plusieurs modèles ont été propo- sés pour quantifier cette érosion [Corbel, 1959; Pulina, 1972; Ford, 1981]. Le modèle d'érosion maximum de White [1984], le plus complet et le plus utilisé, fait l'hypothèse que les eaux d'infiltration sont à l'équilibre avec les carbonates:

$\left.\frac{d z}{d t}\right|_{\text {dissolution }}=D R=-\frac{1000 m_{\mathrm{Ca}}(\bar{P}-E)}{\rho_{\mathrm{CaCO}_{3}}}\left[\mathrm{Ca}^{2+}\right]_{\text {eqopen }}$

où $D R(\mathrm{~m} / \mathrm{s})$ est la vitesse de l'érosion superficielle, $\bar{P}-E(\mathrm{~m} / \mathrm{s})$ est la pluie effective, $m_{\mathrm{Ca}}(\mathrm{kg} / \mathrm{mol})$ est la masse atomique de la calcite et $\rho_{\mathrm{CaCO} 3}\left(\mathrm{~kg} / \mathrm{m}^{3}\right)$ est la masse volumique de la calcite. La concentration du calcium à l'équilibre peut être déterminée analytiquement en fonction des constantes d'équilibre et de la pression du dioxyde de carbone [White, 1988] :

$$
\left[\mathrm{Ca}^{2+}\right]_{\text {eqopen }}=\left(\frac{\mathrm{K}_{\mathrm{I}} \mathrm{K}_{\mathrm{C}} \mathrm{K}_{\mathrm{H}} \mathrm{P}_{\mathrm{CO}_{2} \text { open }}}{4 \mathrm{~K}_{2} \gamma_{\mathrm{Ca}} \gamma_{\mathrm{HCO}_{3}}^{2}}\right)^{\frac{1}{3}}
$$

Les valeurs de $\left[\mathrm{Ca}^{2+}\right]_{\text {eqopen }}$ varient en fonction de la pression en $\mathrm{CO}_{2}$ et de la température.

La relation (4) présente plusieurs intérêts: elle ne nécessite aucun calage et elle combine les trois variables climatiques que sont la température, la pression du dioxyde de carbone et les précipitations. Chacun de ces paramètres possède un degré d'influence différent sur cette érosion. En appliquant le modèle (4), on fait l'hypothèse que l'érosion chimique est uniforme sur l'ensemble du karst. Pour les karsts en cockpit, $D R \approx 1,3 \times 10^{-4} \mathrm{~m} / \mathrm{an}$.

La cinétique de dissolution de la calcite est également bien connue et sera notée F dans la suite de l'article [Buhmann and Dreybrodt, 1985]. 


\section{Concepts du modèle}

La karstification est un processus si complexe qu'il est très difficile d'établir les relations évidentes et quantifiables entre ses composantes: la dissolution de la calcite, les propriétés géologiques des carbonates ou encore les variables climatiques. Il existe une littérature abondante sur la description qualitative de ces processus [e.g. Sweeting, 1972; Williams, 1985, 1987], mais très peu sur les relations quantitatives.

Bien que la dissolution joue un rôle prépondérant dans le développement du karst, ce n'est pas le seul processus. De nombreux auteurs mettent en évidence la relation forte entre l'érosion chimique en surface et les processus de karstification sous-jacents sans toutefois en donner une expression qualitative.

La dissolution dans un réseau de fractures correspond à une érosion chimique de la roche, donc à sa disparition dans les profondeurs du réseau karstique. La disparition de cette quantité de matière entraîne logiquement avec elle l'abaissement de la surface topographique dont le sol vient combler les dépressions. Ceci nous amène donc à formuler une hypothèse sous-tendue par la théorie de l'épikarst: l'érosion superficielle est proportionnelle à la dissolution des fractures de la zone épikarstique.

La théorie de l'épikarst [e.g. Ford and Williams, 1989] apporte les bases descriptives de la formation de certains types de karst, comme les karsts en cockpit: sur une topographie caractérisée par une légère pente initiale et pourvue d'un réseau de fractures, les eaux météoriques s'infiltrent (figures $4 a$ et 4b). Du fait de la rapide réduction de l'ouverture des fractures avec la profondeur, les eaux de la zone subcutanée sont ainsi perchées et s'écoulent des points hauts topographiques vers les points bas topographiques. Les points hauts locaux et les points bas de la "nappe" perchée correspondent globalement à ceux de la topographie susjacente. Ainsi l'écoulement des eaux se fait préférentiellement au niveau des fractures situées aux points bas de la nappe perchée et donc aux points bas de la topographie. La dissolution est donc plus importante au niveau des points bas de la topographie. Apparaît ainsi un feed-back positif entre l'écoulement dans les fractures et la dissolution. Ce feedback positif est entretenu par l'apport d'eau de subsurface au niveau des fractures situées aux points bas de la topographie. Or, cette quantité d'eau qui s'écoule dans les fractures n'augmente pas indéfiniment, le système n'étant alimenté que par les précipitations. Si ce feed-back est très important en début de simulation (i.e. les fractures s'ouvrent très rapidement), on verra sur la figure 13 qu'il diminue fortement avec le temps de simulation.

\section{A. Géométrie du modèle et conditions initiales}

Afin de vérifier cette hypothèse, nous mettons en place dans le modèle CHILD un réseau de fractures à géométrie simple. Initialement, un plateau calcaire est discrétisé en 4600 cellules de Voronoï (figure 5) et disséqué par des fractures planes verticales (figure 6). Les fractures ont une profondeur de $100 \mathrm{~m}$ [Williams, 1985] et une ouverture initiale $a_{0}=10^{-4} \mathrm{~m}$ [Gabrovsek and Dreybrodt, 2001]. Ce réseau de fractures est couvert d'un sol fin et permet d'affecter à chaque cellule de Voronoï une conductivité hydraulique, c'est-à-dire une capacité d'infiltration à l'eau. Les conductivités hydrauliques permettent ainsi de quantifier la part des écoulements de surface et de sub-surface (figure 7). Si la conductivité hydraulique d'une cellule de Voronoï n'est pas suffisante pour que toute l'eau présente s'infiltre (l'eau de pluie + celle venant de l'amont) alors la quantité d'eau restante s'écoule vers la cellule de Voronoï située en aval.

Sur l'ensemble du domaine d'étude sont disposées des fractures espacées de $s=1 \mathrm{~m}$. La conductivité hydraulique obtenue est donc de l'ordre de $10^{-7} \mathrm{~m} / \mathrm{s}$, valeur caractéristique d'une faible karstification initiale [Singhal and Gupta, 1999].

De plus, nous considérerons que les dépressions sont disposées en étoile autour des sommets [e.g. Sweeting, 1972]. Ainsi les écoulements sont initialement dirigés, grâce à une pente de $1 \%$, des sommets vers les dépressions. La distance moyenne entre dépression et sommet est de $110 \mathrm{~m}$ [Lyew-Ayee, 2004].

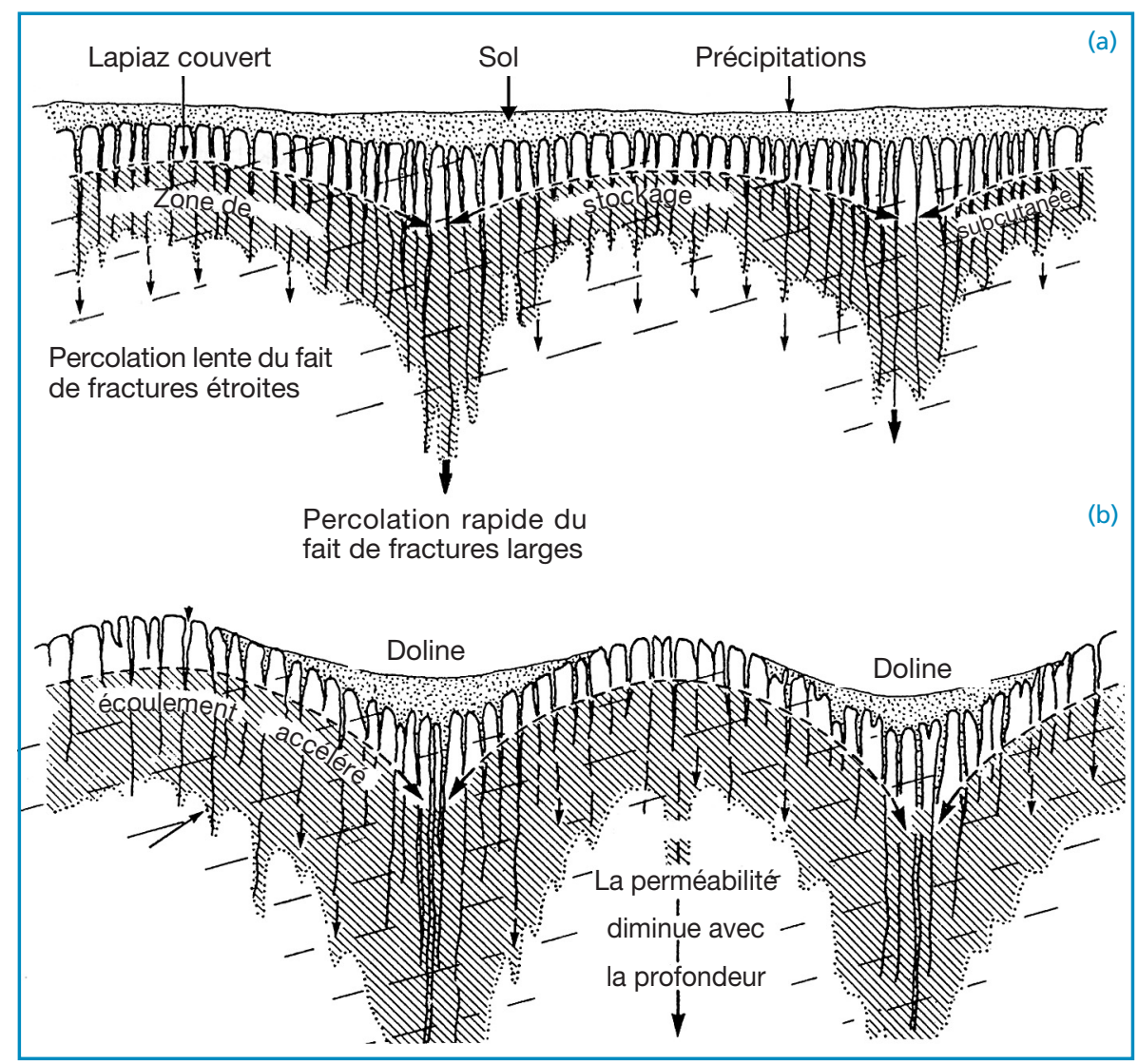

Figure 4: Illustration de la zone épikarstique de carbonates karstifiés [d'après Williams, 1985]. Les fractures permettent aux eaux de s'infiltrer (a) et localisent ainsi la dissolution des carbonates. Dissolution souterraine et évolution de la topographie sont alors fortement corrélées (b).

Illustration of the subcutaneous zone in karstified rocks (after Williams, 1985). The interconnected fissures network provides solute pathways (a). Fissures are considerably widened by solution. Preferred flow paths develop at the base of the epikarstic zone. These are enhanced by solution and become increasingly dominant (b). 


\section{B. Etapes de calcul}

Le détail des procédures de calcul des écoulements dans les fractures et de leur dissolution n'est pas présenté ici pour des raisons de place mais peut être demandé aux auteurs.

Pour synthétiser, la dissolution dans une fracture, l'élargissement de son ouverture qui en découle et l'érosion superficielle sont modélisés successivement par les étapes suivantes:

1. La conductivité hydraulique d'une fracture type et la conductivité hydraulique moyenne de la cellule de Voronoï correspondante sont calculées à partir de la géométrie initiale du système (ouverture et espacement des fractures);

2. Le débit disponible à l'infiltration sur une cellule de Voronoï est alors évalué. On calcule ainsi le débit qui peut circuler dans une fracture en fonction de sa conductivité hydraulique;

3. Si le régime d'écoulement dans les fractures est laminaire, le débit est calculé par l'équation de Poiseuille;

ou

3'. Si le régime hydraulique dans les fractures est turbulent, le débit est calculé par l'équation de Darcy-Weissbach;

4. On calcule la valeur des différents paramètres liés à la dissolution des fractures;

5. On obtient la nouvelle valeur de l'ouverture de la fracture (figure 8), en considérant que la dissolution dans la fracture est uniforme et donnée par le flux de sortie [Dreybrodt et al., 1999] :

$\left.\frac{d a}{d t}\right|_{i}=\frac{m_{C a}}{\boldsymbol{\rho}_{\mathrm{CaCO}}} F(L)$

6. On calcule l'évolution de la topographie associée:

$\left.\frac{d z}{d t}\right|_{i}=\alpha_{i} D R$

où $D R(\mathrm{~m} / \mathrm{s})$ est l'érosion maximale du modèle de White, 1984 définie par l'équation (4) et:

$$
\alpha_{i}=\frac{\left.\frac{d a}{d t}\right|_{i}}{\left.\frac{d a}{d t}\right|_{\max }}
$$

$\left.\frac{d a}{d t}\right|_{\max }(\mathrm{m} / \mathrm{s})$ est la vitesse d'ouverture maximale d'une fracture durant un pas de temps donné. L'évolution de la topographie (équation 7) est basée sur l'hypothèse que nous avons formulée précédemment et qui découle elle-même des processus décrits par la théorie de l'épikarst: il y a correspondance

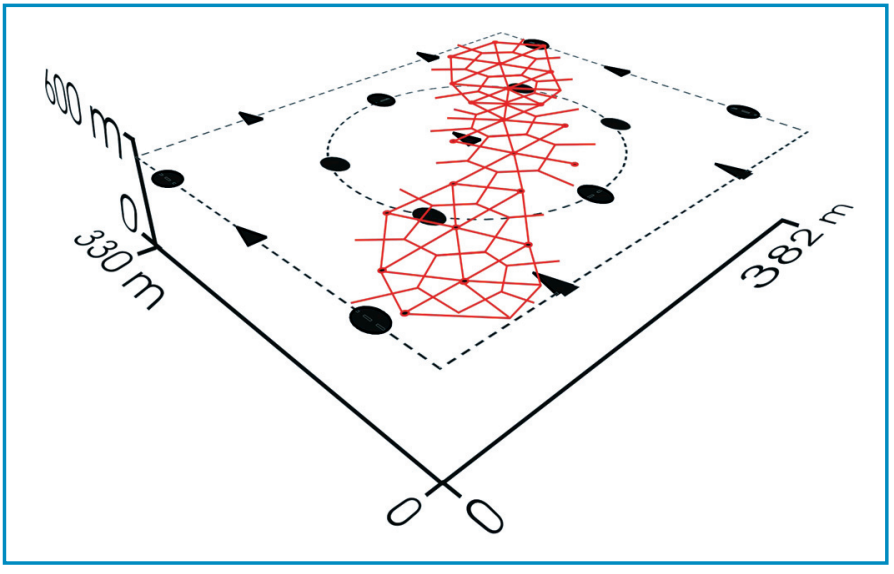

locale entre évolution de la topographie et dissolution des fractures de la zone subcutanée. L'équation (8) montre que l'évolution de la topographie ne peut pas être supérieure à $D R(\mathrm{~m} / \mathrm{s})$, puisque $0 \leq \alpha_{i} \leq 1$. De plus, $\alpha_{i}$ est un facteur directement lié aux processus de dissolution des fractures de la zone épikarstique (écoulement et dissolution). Il permet ainsi d'avoir une évolution de la topographie anisotrope dans l'espace et le temps.

7. On met à jour les valeurs des conductivités hydrauliques des fractures et des cellules de Voronoï à partir des nouvelles valeurs des ouvertures calculées à l'étape 6 ;

8. L'ensemble des processus du modèle ayant été exécutés pendant ce pas de temps, on reprend à l'étape 1 à partir de la géométrie nouvellement obtenue.
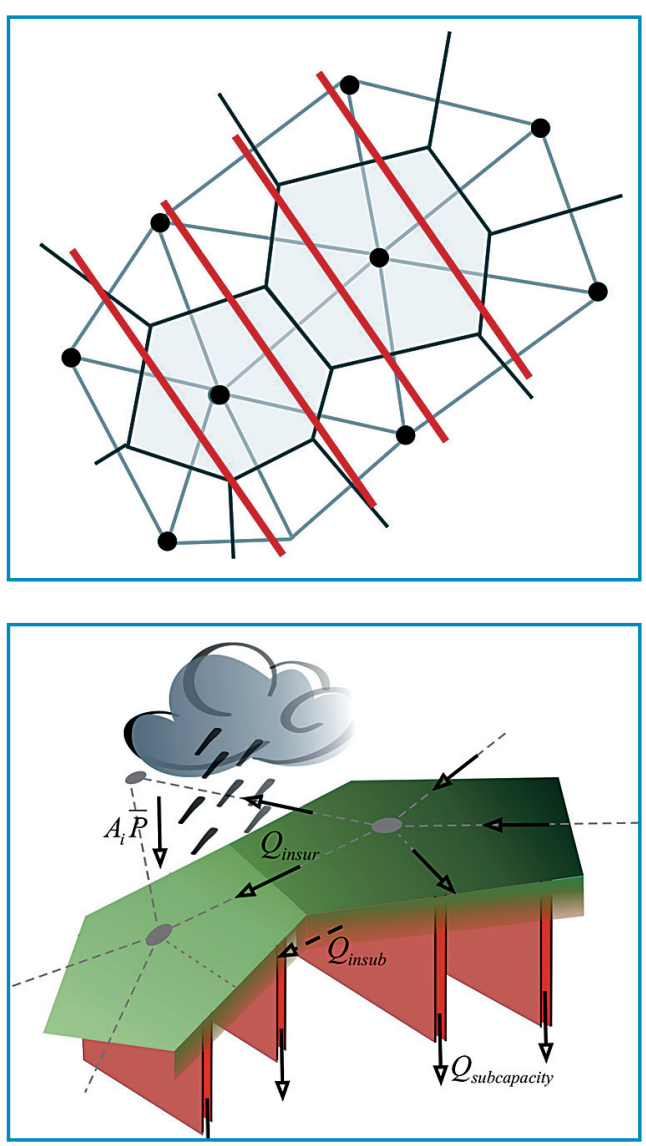

Figure 5: Le domaine d'étude est initialement discrétisé en 4600 cellules de Voronoï (en rouge) qui ont été volontairement grossies ici. Cette discrétisation permet d'avoir un maillage fin de 1 nœud pour $6 \mathrm{~m}$ d'arête. L'eau s'écoule initialement des sommets (triangles noirs) vers les dépressions (disques noirs). The simulated area is discretized into 4600 Voronoï cells (red lines) which provides a thin mesh: 1 node every $6 \mathrm{~m}$. Water initially flows from summits (black triangles) to depressions (black circles).

Figure 6: le domaine d'étude est disséqué par des fractures planes et verticales. Chaque cellule de Voronoï est ainsi associée à une conductivité hydraulique, c'est-à-dire, à une quantité d'eau qui peut potentiellement s'infiltrer. Les fractures étant verticales et parallèles, il n'existe pas d'intersection entre elles. Ce modèle fracturé est donc une simplification de la réalité que I'on rencontre régulièrement dans la littérature [par exemple Bear et al., 1993].

The study area is dissected by vertical smooth fractures. Each Voronoï cell is linked to a hydraulic conductivity. As fractures are vertical and smooth there is no intersection between them.

Figure 7: les écoulements de l'eau souterraine et de surface sur le domaine suivent une procédure simple contrôlée par les conductivités hydrauliques. surface and sub-surface flows are computed according to a simple routing procedure controlled by hydraulic conductivities. 


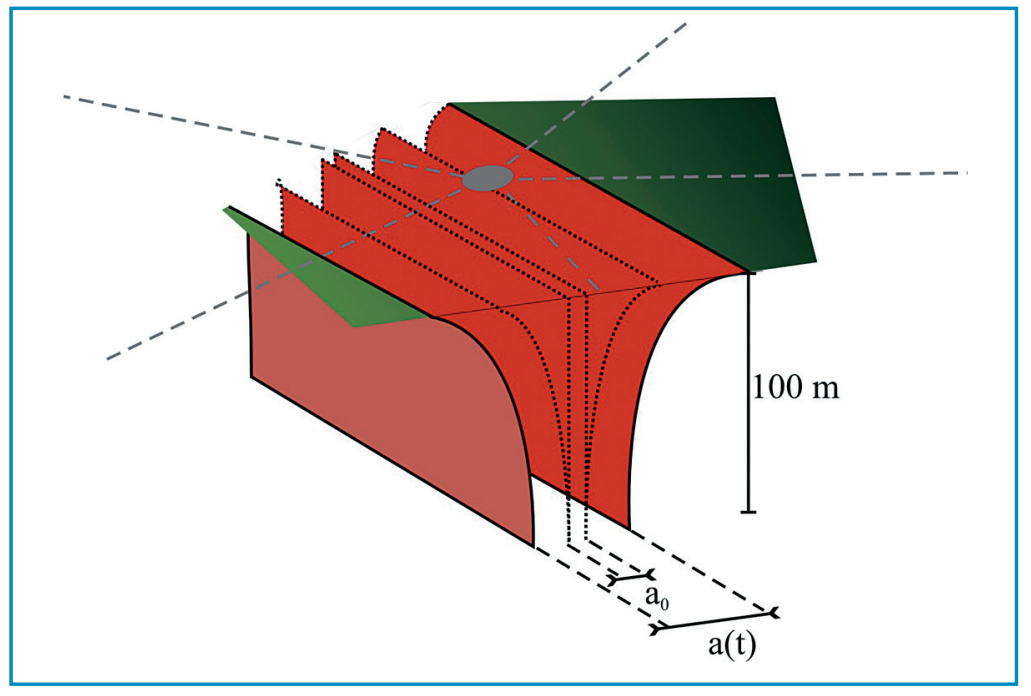

Figure 8: Les fractures sont assimilées à des plans verticaux $\mathrm{d}^{\prime}$ ouverture initiale $10^{-4} \mathrm{~m}$ et de profondeur $100 \mathrm{~m}$. La dissolution

des carbonates provoque une augmentation de l'ouverture de

ces fractures et donc une augmentation de la conductivité

hydraulique: feed-back positif.

Fractures are considered as

vertical planes with initial

aperture $10^{-4} \mathrm{~m}$ and $100 \mathrm{~m}$

length. Due to carbonate dissolution, fracture widening

takes place all along the profile and aperture is enlarged over time producing a positive feed-back.

Figure 9: La structure géométrique initiale est superposée ici à un paysage simulé de cockpit. Les dépressions sont disposées en étoile autour des sommets.

Initial spatial geometry is overlapped with a simulated landscape. Depressions are starsshaped around summits. reproduire les formes moyennes réelles des karsts en cockpit? Si tel est le cas, alors les hypothèses qui sous-tendent le modèle permettront de valider quantitativement une partie des processus de formation des paysages karstiques.

\section{A. Simulation de référence}

Une simulation de référence est préalablement effectuée afin de générer la forme d'un cockpit, sans prise en compte de la dissolution. La dernière composante de l'équation (1) est donc nulle.

Cette simulation de référence demande au préalable une paramétrisation des équations (2) et (3) que nous ne développons pas ici mais qui est décrite en détail dans Bogaart et al., 2003.

Après le dépôt des sédiments calcaires durant l'Oligocène (70 millions d'années avant notre ère) et l'Eocène (45 millions d'années avant notre ère), la région des cockpits (Jamaïque) aurait subi une élévation tectonique l'amenant à une hauteur d'environ $600 \mathrm{~m}$ au-dessus du niveau de la mer [LyewAyee, 2004]. La karstification aurait débuté à la fin du Miocène ou au début du Pliocène, en raison des conditions climatiques favorables [Pfeffer, 1997]. Ceci nous permet de simplifier nos équations en imposant la valeur de $\frac{d z}{d t}$ $\left.\frac{d z}{d t}\right|_{\text {tectonique }}=0 \mathrm{~m} / \mathrm{s}$ dans l'équation (1) et de faire l'hypothèse que la néo-tectonique ne joue aucun rôle dans la karstification actuelle. De nombreuses études ont été faites sur les processus de transfert des sédiments sur les versants [e.g. Martin et Church, 1997], et la valeur de la diffusivité pour des processus lents et continus donne $10^{-4} \leq k_{D} \leq 10^{-2} \mathrm{~m}^{2} / \mathrm{an}$ (équation 2). Comme nous l'avons dit précédemment, la valeur du coefficient de diffusion est dépendante de la nature de la roche et du climat. Dans notre cas, nous attendions plutôt des valeurs faibles de $k_{D}$, les roches carbonatées étant caractérisées comme très dures. Devant le manque d'information sur sa valeur, nous testerons plusieurs valeurs de $k_{D}$.

Cinq simulations ont été menées en faisant varier la pluie annuelle $<P>(\mathrm{m} / \mathrm{an})$ et le coefficient de diffusion $k_{D}\left(\mathrm{~m}^{2} / \mathrm{an}\right)$. Les simulations ont une durée de 10 millions d'années afin de couvrir la fin du Miocène, l'ensemble du Pliocène et une partie du Pléistocène; sachant que l'ensemble des théories admet que la formation des karsts en cockpit s'est déroulée essentiellement durant le Pliocène [Pfeffer, 1997]. Tous les 10000 ans la pente moyenne et le relief relatif du cockpit témoin sont calculés afin de pouvoir enregistrer son évolution. Nous pouvons ainsi comparer ces valeurs simulées 
avec les mesures réelles moyennes d'un cockpit représentatif de cette région de Jamaïque.

Les résultats montrent (figure 10) qu'à valeur constante du $k_{D}$, l'augmentation des précipitations moyennes améliore les simulations. Toutefois, cette augmentation ne permet pas de tendre vers une relation: pente moyenne - relief relatif, identique à celle observée dans la réalité. En effet la pente moyenne augmente trop vite par rapport au relief relatif.

Avec une valeur constante pour la pluie, nous avons fait varier le coefficient de diffusion $k_{D}$ (figure 10) afin de déterminer l'ordre de grandeur de ce coefficient que nous ne connaissions pas a priori. Comme nous le supposions, connaissant la dureté des carbonates blancs et le climat humide de la Jamaique, il semble que $k_{D}=10^{-3} \mathrm{~m}^{2} / \mathrm{an}$ soit une valeur cohérente par rapport aux valeurs déterminées sur le terrain.

Sur la figure 10, on voit que les courbes résultats ( 1 et 2 par exemple), donnant l'évolution du relief relatif en fonction de la pente moyenne du cockpit, sont plus ou moins longues. Ces différentes longueurs ne doivent par être interprétées par le lecteur comme autant de temps de simulation différents. L'arrêt d'une courbe correspond au fait qu'un équilibre a été atteint. Ainsi, si nous prenons l'exemple de la courbe 2 sur la figure 10 , on constate qu'au bout d'un temps $t_{2}$, le système relief relatif - pente est constant, c'est-à-dire qu'il garde quasiment la même valeur quel que soit le temps $t \geq t_{2}$. En prenant maintenant la courbe 1 de la figure 10, on voit que la simulation correspondante amène également à un équilibre mais vers des valeurs plus faibles du système relief relatif pente, ce qui est cohérent puisque pour la simulation 1 , les précipitations sont en moyenne deux fois plus faibles que pour la simulation 2 .

Ces simulations de référence apportent une conclusion importante: quelles que soient les valeurs des paramètres du modèle, il n'est pas possible de reproduire la morphométrie d'un cockpit ayant les caractéristiques moyennes de ceux observés en réalité. L'érosion mécanique et la diffusion sédimentaire ne sont donc pas les seuls processus à prendre en compte.

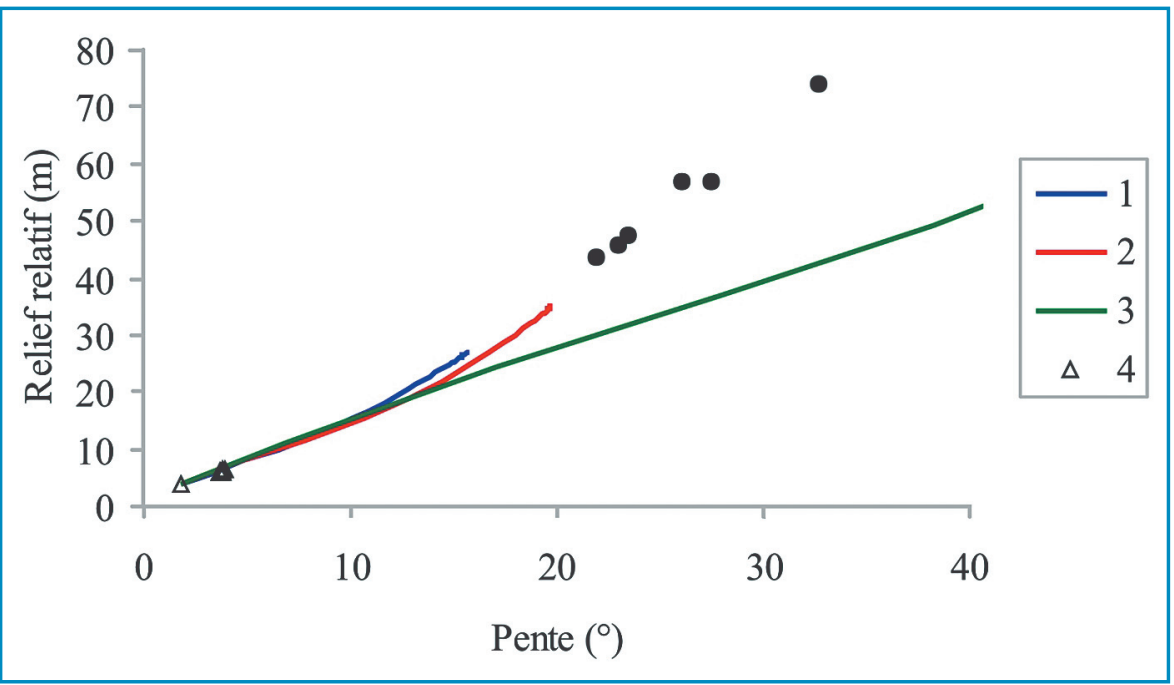

Figure 10: Résultats de la simulation de référence. Les données réelles de Leyw-Ayee, 2004 sont représentées par des disques noirs et indiquent la relation entre la pente moyenne et le relief relatif.

$1:\langle P\rangle=1 \mathrm{~m} / \mathrm{an}$ et $\mathrm{k}_{\mathrm{D}}=10^{-3} \mathrm{~m}^{2} / \mathrm{an} \quad 2:\langle\mathrm{P}\rangle=2 \mathrm{~m} / \mathrm{an}$ et $\mathrm{k}_{\mathrm{D}}=10^{-3} \mathrm{~m}^{2} / \mathrm{an}$

$3:\langle\mathrm{P}\rangle=2 \mathrm{~m} / \mathrm{an}$ et $\mathrm{k}_{\mathrm{D}}=10^{-4} \mathrm{~m}^{2} / \mathrm{an} \quad 4:\langle\mathrm{P}\rangle=2 \mathrm{~m} / \mathrm{an}$ et $\mathrm{k}_{\mathrm{D}}=10^{-2} \mathrm{~m}^{2} / \mathrm{an}$

Results of the reference simulations Real data from Lyew-Ayee's study 2004 are black circles and represent average values of both the relative relief and the slope in the cockpit country, Jamaica.

\section{B. Simulations avec le modèle de dissolution anisotrope}

Il n'est pas facile de déterminer la valeur de la pression en $\mathrm{CO}_{2}$ d'un sol, cette dernière variant avec de nombreux paramètres parmi lesquels l'humidité ou l'activité biologique. L'ordre de grandeur de cette pression dans les sols des cockpits à Puerto Rico est de $0,01 \leq P_{\mathrm{CO} 2}$ $\leq 0,1 \mathrm{~atm}$. [Miotke, 1975 in White, 1984, p. 195]. Cette valeur correspond également aux observations de Smith and Atkinson [1976] sur la région des cockpits en Jamaïque $\left(P_{\mathrm{CO} 2} \approx 0,017\right.$ atm.). Cette valeur de la pression en dioxyde de carbone correspond à l'ordre de grandeur pour un système ouvert, en contact direct avec l'atmosphère. Pour ce qui est d'un système fermé, comme une fracture, la valeur admise est bien plus faible, de l'ordre de $10^{-3}$ atm [Dreydbrot, 1985; Romanov et al., 2003; Kaufman and Braun, 2001].

Les variables environnementales étant soit déterminées en fonction de celles données dans la bibliographie, soit fixées aux valeurs les plus cohérentes en fonction des simulations précédentes $\left(<P>\right.$ et $\left.k_{D}\right)$, nous n'avons que peu de marche de manœuvre sur la simulation. Les seuls paramètres que nous pouvons modifier sont la concentration d'entrée du calcium dans les fractures $\left(\left[\mathrm{Ca}^{2+}\right]_{\text {inclose }}\right)$, la durée de la pluie $\left(\bar{T}_{r}\right)$ et le temps entre les pluies $\left(\bar{T}_{b}\right)$. $\left[\mathrm{Ca}^{2+}\right]_{\text {inclose }}$ dépend essentiellement des caractéristiques du sol sus-jacent aux fractures. La valeur de $\left[\mathrm{Ca}^{2+}\right]_{\text {inclose }}$ est nulle si aucun sol n'est présent au-dessus de la fracture. Sa valeur augmente avec l'épaisseur du sol. En effet, plus le sol est épais et plus les eaux qui s'y infiltrent ont le temps de se charger en calcium.

De nombreux auteurs [e.g. LyewAyee, 2004] décrivent un sol très fin dans la région des cockpits de Jamaïque. Pour cette raison, nous faisons l'hypothèse que $\left[\mathrm{Ca}^{2+}\right]_{\text {inclose }}=0 \mathrm{~mol} / \mathrm{m}^{3}$, l'eau d'infiltration n'ayant pas le temps d'atteindre la concentration à l'équilibre. Le rapport $\frac{\bar{T}_{r}+\bar{T}_{b}}{\bar{T}_{r}}$ peut être considéré comme un facteur climatique caractérisant l'intermittence des pluies [Tucker and Bras, 2000]. Dans le modèle nous ferons varier ce facteur de 1 à 50. Plus le rapport augmente et plus les pluies sont espacées dans le temps et vice versa.

L'utilisation de ce modèle peut permettre de valider numériquement la théorie de l'épikarst, théorie descriptive de la morphométrie des karsts en cockpit [Williams, 2004]. Les conditions aux limites et les conditions initiales sont ici les mêmes que dans les simulations précédentes à l'exception du réseau de fractures. En effet, comme nous l'avons décrit précédemment, un réseau de fractures est généré afin de reproduire les conditions hydrodynamiques proches de la réalité : partage entre écoulements de surface et de sub-surface. 


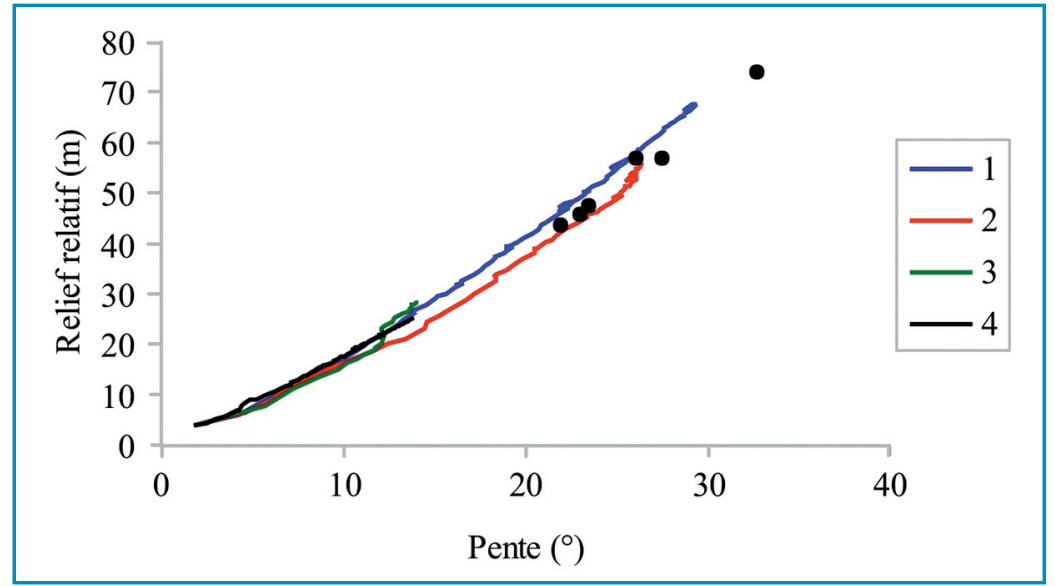

Figure 11: Résultats des simulations avec le modèle de dissolution anisotrope. Pour toutes les simulations, $k_{D}=10^{-3} \mathrm{~m}^{2} / \mathrm{an}$. Les disques noirs ont la même signification que sur la figure 10 .

$$
\begin{aligned}
& 1:\left\langle\mathrm{P}>=2 \mathrm{~m} / \mathrm{an}, \bar{T}_{r}=1000 \text { ans, } \bar{T}_{b}=0 \text { an }\left(\frac{\bar{T}_{r}+\bar{T}_{b}}{\bar{T}_{r}}=1\right)\right. \\
& 2:<\mathrm{P}>=2 \mathrm{~m} / \mathrm{an}, \bar{T}_{r}=500 \text { ans, } \bar{T}_{b}=\quad 500 \text { ans }\left(\frac{\bar{T}_{r}+\bar{T}_{b}}{\bar{T}_{r}}=2\right) \\
& 3:<\mathrm{P}>=2 \mathrm{~m} / \mathrm{an}, \bar{T}_{r}=100 \text { ans, } \bar{T}_{b}=900 \text { ans }\left(\frac{\bar{T}_{r}+\bar{T}_{b}}{\bar{T}_{r}}=10\right) \\
& 4:<\mathrm{P}>=2 \mathrm{~m} / \mathrm{an}, \bar{T}_{r}=20 \text { ans, } \bar{T}_{b}=980 \text { ans }\left(\frac{\bar{T}_{r}+\bar{T}_{b}}{\bar{T}_{r}}=50\right)
\end{aligned}
$$

Results of the simulations using the anisotropic dissolution model $\left(k_{D}=10^{-3} \mathrm{~m}^{2} / \mathrm{an}\right)$.

La dissolution souterraine résulte de l'infiltration de l'eau dans les fractures et dépend ainsi de la position des fractures le long de la pente topographie. Plus les fractures sont situées à l'aval de la pente et plus elles reçoivent une grande quantité d'eau de la nappe perchée [Williams, 2004], et plus la dissolution est importante, et vice versa. Ce processus est rendu possible parce que nous avons fait l'hypothèse précédemment que l'équilibre cinétique n'est jamais atteint du fait de la dynamique rapide de l'eau dans les fractures. Apparaît ainsi un feed-back positif entre l'écoulement dans les fractures et la dissolution, car plus la dissolution est importante, plus la fracture s'ouvre et donc plus elle laisse s'infiltrer l'eau. En théorie, ce feed-back positif amène à un

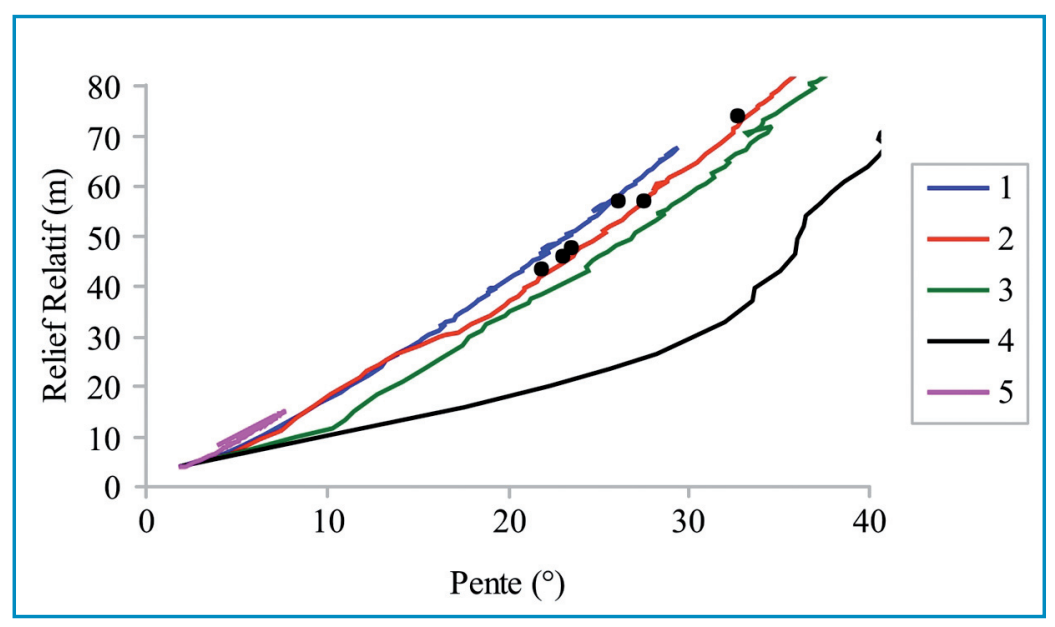

Figure 12: Résultats des simulations avec le modèle de dissolution anisotrope. Les disques noirs ont la même signification que sur la figure 10 .

$$
\begin{aligned}
& \text { Pour toutes les simulations, }\langle\mathrm{P}\rangle=2 \mathrm{~m} / \mathrm{an} \text { et } \frac{\bar{T}_{r}+\bar{T}_{b}}{\bar{T}_{r}}=1 \text {. } \\
& 1: \mathrm{k}_{\mathrm{D}}=10^{-3} \mathrm{~m}^{2} / \mathrm{an} \\
& \text { 4: } \mathrm{k}_{\mathrm{D}}=10^{-4} \mathrm{~m}^{2} / \mathrm{an} \\
& 2: \mathrm{k}_{\mathrm{D}}=8 \cdot 10^{-4} \mathrm{~m}^{2} / \mathrm{an} \\
& 5: k_{D}=5 \cdot 10^{-3} \mathrm{~m}^{2} / a n \\
& 3: \mathrm{k}_{\mathrm{D}}=5 \cdot 10^{-4} \mathrm{~m}^{2} / \mathrm{an}
\end{aligned}
$$

Results of the simulations using the anisotropic dissolution model. comportement extrême du système fracturé: l'augmentation exponentielle de l'ouverture des fractures [Dreybrodt, 1985]. Cette augmentation n'est entretenue que pour des conditions hydrodynamiques particulières, on parle de conditions aux limites à potentiel imposé: la quantité d'eau qui entre dans le système fracturé est constante et en quantité toujours suffisante. Dans la réalité, ce feed-back positif est modéré par des conditions hydrodynamiques à l'entrée du système fracturé qui sont très variables et dépendantes des aléas climatiques.

Les résultats des simulations du modèle anisotrope de la dissolution sont présentés sur les figures 11 et 12. Dans un premier temps on peut dire que ce modèle simule des karsts en cockpit dont les propriétés morphométriques sont très proches de celles observées dans la réalité. Ainsi l'adéquation entre la simulation 1 et les données réelles de Lyew-Ayee [2004] vont dans le sens d'une confirmation numérique de la théorie de l'épikarst.

Au début des simulations, l'ouverture est la même pour toutes les fractures $\left(a_{0}=10^{-4} \mathrm{~m}\right)$, quelle que soit leur position par rapport au sommet. Les eaux de surface et de sub-surface s'écoulant des sommets vers les dépressions, la quantité d'eau pouvant s'infiltrer dans les fractures est plus importante en aval. Ainsi, la dissolution des parois des fractures aval est plus importante que celles situées en amont (figure 13). En conséquence, l'érosion étant corrélée à la dissolution des fractures, elle aussi plus forte en aval (figure 14).

\section{Discussion et conclusion}

L'objectif de cette étude sur la morphologie des karsts en cockpit était de simuler les processus de leur évolution à partir d'hypothèses sur leur genèse. Notre modèle est basé sur une dissolution anisotrope dans l'espace et le temps conformément aux processus décrits dans la théorie de l'épikarst.

Les simulations du modèle sont comparées à des simulations de référence où seule l'érosion mécanique est envisagée et où la dissolution chimique des carbonates est donc absente.

Des karsts simulés, on peut extraire les caractéristiques morphologiques qui évoluent en fonction du temps. Deux caractéristiques morphologiques ont été choisies, au regard des travaux de Lyew- 
Ayee, 2004, pour comparer les karsts simulés aux karsts réels: la pente moyenne et le relief relatif.

L'adéquation entre les morphologies des karsts simulés et réels est une étape essentielle pour valider leurs hypothèses de genèse. Toutefois c'est une étape nécessaire mais probablement pas suffisante.

L'érosion karstique met en place des processus divers et complexes que nous n'abordons pas ici, par exemple l'entraînement des particules vers le fond des fractures ou l'écroulement des zones fracturées dans des cavités sous-jacentes.

Ainsi, cette étude nous a permis de mettre en évidence l'importance de la prise en compte des processus d'anisotropie temporelle et spatiale dans le cas de l'évolution morphométrique des karsts en cockpit.

L'anisotropie spatiale correspond à l'hétérogénéité des valeurs de conductivités hydrauliques fréquemment observées dans les systèmes karstiques. Cette hétérogénéité participe à la différenciation spatiale de l'hydraulique de surface et de l'hydraulique souterraine et modifie ainsi fortement les comportements chimiques de dissolution de la roche.

L'anisotropie temporelle rend compte des effets de cinétique chimique dans les processus de dissolution. La prise en compte de la cinétique est indispensable pour modéliser la dissolution des milieux carbonatés fracturés.

Etant donné les résultats, nous pouvons mettre en avant un certain nombre de points: - Seule, l'érosion mécanique n'est pas une hypothèse suffisante pour expliquer la forme des karsts en cockpit. Ceci est tout à fait cohérent, sachant que de nombreux auteurs rapportent l'importance des processus de dissolution par rapport à l'érosion pour ce qui concerne les systèmes karstiques.

- En considérant que la dissolution souterraine est également un facteur important dans l'évolution de la géomorphologie superficielle du karst, ce que traduit la théorie de l'épikarst, on obtient effectivement des simulations très proches de la réalité. Le modèle de dissolution spatiotemporelle anisotrope traduit le fait que les fractures les plus en aval sur la pente reçoivent plus d'eau d'infiltration de la nappe perchée (zone épikarstique), et vice versa. Ainsi, les parois des fractures les plus en aval s'agrandissent plus et ces fractures laissent donc infiltrer plus d'eau, et ainsi de suite. Ce feed-back positif entre écoulement et dissolution est la pierre angulaire de nombreuses théories sur le fonctionnement des systèmes karstiques. Or, plus la dissolution est active dans la zone subcu-

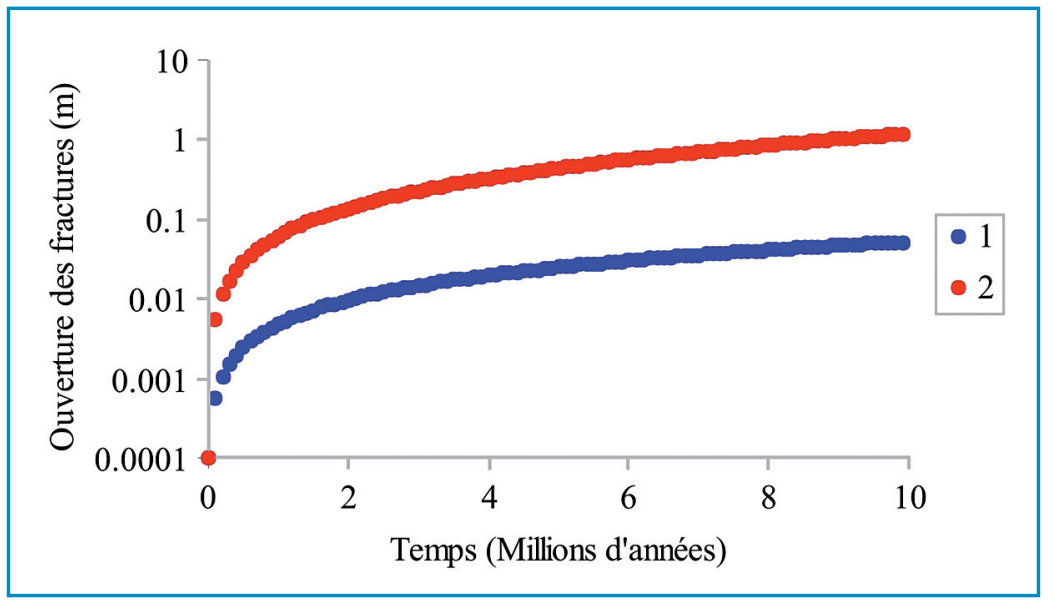

Figure 13: La dissolution des parois des fractures et donc l'augmentation de leur ouverture dépend de leur position sur la pente du cockpit. La fracture 1 est située à $20 \mathrm{~m}$ du sommet alors que la fracture 2 se trouve, elle, $60 \mathrm{~m}$ plus en aval. L'écoulement de sub-surface est donc plus important dans la fracture 2 , la dissolution y est donc plus importante.

Dissolution of fractures depends on their locations on the cockpit slope. Fracture 1 is located at $20 \mathrm{~m}$ from the summit and fracture 2 is $60 \mathrm{~m}$ farther. Subsurface flow is thus greater in fracture 2, so dissolution is greater as well. Fracture widenning 2 is then greater by several orders of magnitude over time.

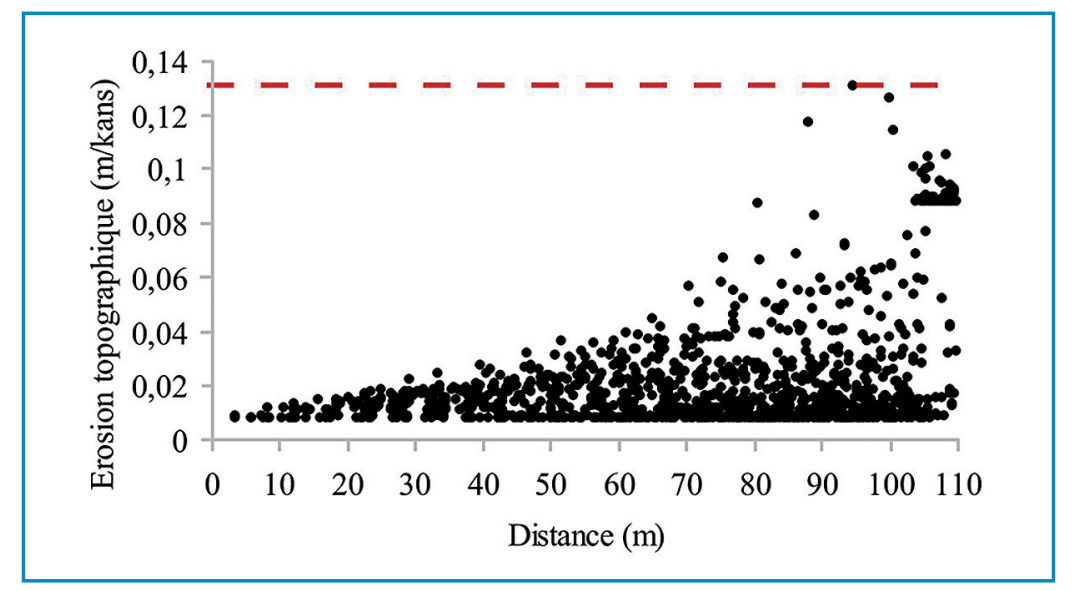

tanée, donc proche de la surface, et plus cette dernière sera érodée.

- Dans le modèle, les valeurs de la pluie moyenne annuelle varient de $1 \mathrm{~m} / \mathrm{an}$ à $2 \mathrm{~m} / \mathrm{an}$. Les résultats montrent clairement que ces pluies doivent être bien supérieures à $1 \mathrm{~m} / \mathrm{an}$ pour simuler des karsts en cockpit réalistes. Ceci est en accord avec les conclusions de nombreux auteurs comme par exemple Sweeting [1972].

- Le facteur climatique $\frac{\bar{T}_{r}+\bar{T}_{b}}{\bar{T}_{r}}$ illustre l'intermittence des pluies à l'échelle géologique. Ce facteur tend vers 1 lorsque la fréquence des pluies augmente. Les résultats montrent un fort impact de ce facteur sur les simulations. Aussi, il semble que l'on ne puisse reproduire la morphologie des karsts que pour des pluies très intenses et très fréquentes.

- Le coefficient de diffusion $k_{D}$ influence aussi fortement les simulations. Toutefois, les
Figure 14: I'érosion chimique est anisotrope sur la pente d'un cockpit. Cette érosion est plus importante en aval (la distance entre sommet et dépression est de l'ordre de $110 \mathrm{~m}$ ). La ligne rouge représente la valeur du modèle de White, 1984, DR (équation 4) Denudation rate is anisotropic on the cockpit slope. Denudation is more important downtream (distance from a summit to a depression is $110 \mathrm{~m}$ ). The red line is the maximum denudation rate of the White's model, 1984. 
paysages simulés se rapprochent des paysages réels seulement pour un mince intervalle de valeurs autour de $k_{D} \approx 10^{-3} \mathrm{~m}^{2} / \mathrm{an}$.

Pour comparer les paysages simulés et les paysages réels, nous avons utilisé deux paramètres morphométriques: la pente et le relief relatif. Or, il existe bien d'autres méthodes d'analyse des paysages et en particulier celle des caractéristiques scalantes [Weissel et al., 1994], qui semble être une méthode particulièrement bien adaptée aux cas des karsts en cockpit [Lyew-Ayee, 2004]. De plus, nous avons étudié les processus sur un seul cockpit. Maintenant il serait important de tester l'influence des paramètres lors d'un changement d'échelle, en simulant un paysage entier de karst en cockpit (figure 14) et en analysant ses caractéristiques morphologiques.

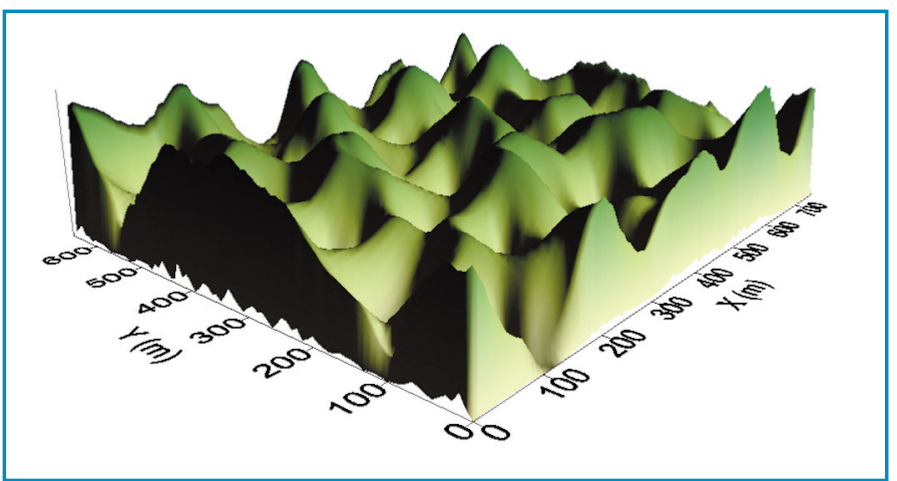

Figure 15: Paysage simulé d'un karst en cockpit. Il est intéressant de comparer cette vue virtuelle avec la figure 1 A simulated cockpit karst landscape. It is interesting to compare this artifical view with a real one on Figure 1.

\section{Bibliographie}

BEAR J., TSANG C.F. and DE MARSILY G., 1993 - Flow and contaminant transport in fractured rock, Academic Press, $230 \mathrm{p}$.

BEAUMONT C., FULLSACK P. and HAMILTON J., 1992 - Erosional control of active compressional orogens. In: McClay K. R. (Ed.), Thrust Tectonics. New York: Chapman and Hall, pp. 1-18. BOGAART P.W., TUCKER G.E. and DE VRIES J.J., 2003 - Channel network morphology and sediment dynamics under alternating periglacial and temperate regimes: a numerical simulation study, Geomorphology, 54 (3-4), pp. 257-277.

BRAUN J. and SAMBRIDGE M., 1997

- Modelling landscape evolution on geological time scales: a new method based on irregular spatial discretization, Basin Research, 9, pp. 27-52.

BROOK G.A. and HANSON M., 1991

Double fourier series analysis of cockpit and doline and karst near browns town, Jamaica, Physical Geography, 12 (1), pp. 37-54.

BUHMANN D. and DREYBRODT W., 1985 - The kinetics of calcite dissolution and precipitation in geologically relevant situations of karst areas. 1. Open system, Chem. Geol. 48, pp. 189-211.

CORBEL J., 1959 - Vitesse de l'érosion, Zeitschrift für Geomorphologie, pp. 31-28.

COULTHARD T.J., 2001 - Landscape evolution models: a software review, Hydrological Processes, 15 (1), pp. 165-173.

COULTHARD T.J., KIRKBY M.J. and MACKLIN M.G., 1998 - Modelling the 1686 flood of Cam Gill Beck, Starbotton. upper Warfedale. In The Quaternary of the Eastern Yorkshire Dales: Field Guide, Howard A, Macklin MG (eds). Quaternary Research Association: London, pp. 11-18.

DAY M.J., 1979 - The hydrology of polygonal karst depressions in northern Jamaica, Zeitsch. Geomorph. N.F., 32, pp. 25-34.
DREYBRODT W., 1985 - Processes in Karst Systems, Springer, Berlin, 240 p. DREYBRODT W., GABROVSEK F. and SIEMERS J., 1999 - Dynamics of the early evolution of karst, in Karst Modelling, Palmer and Palmer Ed., $256 \mathrm{p}$.

EAGLESON P.S., 1978 - Climate, soil, and vegetation. 1. Introduction to water balance dynamics, Water Resour. Res., 14, pp. 705-712.

ENZEL Y., AMIT R., PORAT N., ZILBERMAN E. and HARRISON B.J., 1996 Estimating the ages of fault scraps in the Arava, Israel, Tectonophysics, 253 pp. 305-317.

FORD D.C. and WILLIAMS P.W., 1989 - Karst geomorphology and hydrology, Unwin Hyman, London, 320 p.

FORD D.C., 1981 - Geologic Structure and a New Exploration of Limestone Cavern Genesis. Transactions of the Cave Research Groups of Great Britain, 13, pp. 81-94.

GABROVSEK F. and DREYBRODT W., 2001 - A comprehensive model of the early evolution of karst aquifers in limestone in the dimensions of length and depth, J. Hydrol. 240 3-4, pp. 206-224.

GRUND D., 1914 - The geographical cycle in the karst, Benchmark Papers in Geology, 59, pp. 54-59.

KAUFMANN G. and BRAUN J., 2001

- Modelling karst denudation on a synthetic landscape, Terra Nova, 13 (5), pp. 313-320.

LYEW-AYEE P., 2004 - Digital topographic analysis of cockpit karst: a morphogeological study af the cockpit country region, Jamaica, Ph.D. Thesis, University of Oxford, $210 \mathrm{p}$.

MCKEAN J.A., DIETRICH W.E. FINKEL R.C., SOUTHON J.R. and CAFFEE M.W., 1993 - Quantification of soil production and downslope creep rates from cosmogenic. Geology, 21, pp. 343-346.

MARTIN Y. and CHURCH M., 1997

Diffusion in landscape development models: on the nature of basic trans- port relations, Earth Surface Processes and Landforms 22, pp. 273-279.

PFEFFER K.H., 1997 - Paleoclimate and tropical karst in the west Indies, Zeitsch. Geomorph. N.F., Suppl. B-d 108, pp. 5-13. PULINA M., 1972 - A comment on present day chemical denudation in Poland, Geographica Polonica, 23, pp. 45-62.

ROMANOV D., GABROVSEK F. and DREYBRODT W., 2003 - The impact of hydrochemical boundary conditions on the evolution of limestone karst aquifers. J. Hydrol., 276, pp. 240-253. SINGHAL B. and GUPTA R., 1999 Applied Hydrogeology of Fractured Rocks. Kulwer Academic Press, Dordrecht, The Netherlands. 400 p. SMITH D.I. and ATKINSON T.C., 1976 - Process, landforms and climate in limestone regions, in Derbyshire $\mathrm{Ed}$ Geomorphology and climate. Pp. 367 409. Willey, London.

SWEETING M.M., 1972 - Karst Landforms. MacMillan, London, $150 \mathrm{p}$ TUCKER G.E., LANCASTER S.T. GASPARINI N.M. and BRAS R.E. 2001a - The channel-hillslope integrated landscape development mode (CHILD). In: R.S. Harmon and W.W. Doe, Editors, Landscape Erosion and Evolution Modeling, Kluwer Publishing, Dordrecht, pp. 349-388.

TUCKER G.E., LANCASTER S.T. GASPARINI N.M., BRAS R.E. and RYBARCZYK S.M., 2001b - An objectoriented framework for hydrologic and geomorphic modeling using triangulated irregular networks, Computers and Geosciences 27 8, pp. 959-973.

TUCKER G.E. and BRAS R.E., 2000 - A stochastic approach to modeling the role of rainfall variability in drainage basin evolution, Water Resource Research 36 7, pp. 1953-1964. TUCKER G.E., GASPARINI N.M., BRAS R.E. and LANCASTER S.T., 1999 - $A$ 3D Computer Simulation Model of Drainage Basin and Floodplain Evolution: Theory and Applications, Technical report prepared for U.S. Army
Corps of Engineers Construction Engineering Research Laboratory, pp 125-156.

TUCKER G.E. and SLINGERLAND R.L., 1994 - Erosional dynamics, flexural isostasy, and long-lived escarpments: a numerical modeling study, Journal of Geophysical Research 99, pp. 12229 12243.

VERSTAPPEN H., 1964 - Karst morphology of the Star Mountains and its relation to lithology and climate, Zeitschrift für Geomorphologie, 8 , pp. 40-49.

VERSTEEG H.K. and MALALASEKERA W., 1995 - An Introduction to Computational Fluid Dynamics; The Finite Volume Method. Longman, New York, $257 \mathrm{p}$.

WEISSEL J.K., PRATSON L.F. and MALINVERNO A., 1994 - The length scaling properties of topography, J. Geophys. Res., 99 (B7), pp. 13,99714,012 .

WHITE W.B., 1984 - Rate processes: chemical kinetics and karst landform development, La Fleur RG (ed.). Groundwater as a geomorphic agent. Allen and Unwin, London Boston Sydney pp. 227-248.

WHITE W.B., 1988 - Geomorphology and hydrology of karst terrains, Oxford University Press, New York, $465 \mathrm{p}$.

WILLGOOSE G.R., BRAS R.L. and RODRIGUEZ-ITURBE I., 1991 - A physically based coupled network growth and hillslope evolution model, 1, theory, Water Resources Research 27, pp. 1671-1684.

WILLIAMS P.W., 1985 - Subcutaneous hydrology and the development of doline and cockpit karst, Zeitsch. Geomorph. N.F., 29 4, pp. 463-482.

WILLIAMS P.W., 1987 - Geomorphic inheritance and the development of tower karst, Earth Surface Processes and Landforms, 12, pp. 453-465. WILLIAMS P.W., 2004 - Dolines, in Gunn, J (ed.), Encyclopedia of Caves and Karst Science. Fitzroy Dearborn: New York, pp 304-310. 